\title{
Stratified bundles and étale fundamental group
}

\author{
HÉlÈne ESNAUlt AND XiaOtaO Sun
}

\begin{abstract}
For a smooth projective variety $X$ over an algebraically closed field of characteristic $p>0$, we show that all irreducible stratified bundles on $X$ have rank 1 if and only if the commutator $\left[\pi_{1}, \pi_{1}\right]$ of the étale fundamental group $\pi_{1}$ of $X$ is a pro- $p$-group, and we prove that the category of stratified bundles is semi-simple with irreducible objects of rank 1 if and only if $\pi_{1}$ is Abelian without $p$-power quotient. This answers positively a conjecture by Gieseker [4, page 8].
\end{abstract}

Mathematics Subject Classification (2010): 14F35 (primary); 14G17, 14 G99 (secondary).

\section{Introduction}

Let $X$ be a smooth projective variety defined over an algebraically closed field $k$ of characteristic $p>0$. In [4], stratified bundles are defined and studied. It is shown that they are a characteristic $p>0$ analogue to complex local systems over smooth complex algebraic varieties. In particular, Gieseker shows [4, Theorem 1.10]:

(i) If every stratified bundle is trivial, then $\pi_{1}$ is trivial;

(ii) If all the irreducible stratified bundles have rank 1 , then $\left[\pi_{1}, \pi_{1}\right]$ is a pro- $p$ group;

(iii) If every stratified bundle is a direct sum of stratified line bundles, then $\pi_{1}$ is Abelian without non-trivial $p$-power quotients.

Here $\pi_{1}$ is the étale fundamental group based at some geometric point. He conjectures that in the three statements, the "if " can be replaced by "if and only if". The aim of this note is to give a positive answer to Gieseker's conjecture (see Theorem 3.9).

The converse to (i) is the main result of [3], and it is analogous to the MalcevGrothendieck theorem $([5,10])$ asserting that if the étale fundamental group of a

The first author is supported by the SFB/TR45 and the ERC Advanced Grant 226257. The second author is supported by NBRPC 2011CB302400, NSFC60821002/F02 and NSFC 10731030.

Received December 20, 2011; accepted in revised form August 13, 2012. 
smooth complex projective variety is trivial, then there is no non-trivial bundle with a flat connection.

The complex analogue to the converse to (iii) relies on the following. If $X$ is an Abelian variety over a field $k$, Mumford [11, Section 16] showed that a non-trivial line bundle $L$ which is algebraically equivalent to 0 fulfills $H^{i}(X, L)=0$ for all $i \geq 0$. If $k=\mathbb{C}$, the field of complex numbers, $L$ carries a unitary flat connection with underlying local system $\ell$, and $H^{1}(X, L)=0$ implies that $H^{1}(X, \ell)=0$. In fact, more generally, if $X$ is any complex manifold with Abelian topological fundamental group, and $\ell$ is a non-trivial rank 1 local system, then $H^{1}(X, \ell)=0$ (see Remark 3.11).

Finally the complex analogue to the converse to (ii) and (iii) together says that if $X$ is a smooth complex variety then all irreducible complex local systems on $X$ have rank 1 if and only if $\pi_{1}$ is Abelian. However, the semi-simplicity statement has a different phrasing: all irreducible complex local systems on $X$ have rank 1 and the category is semi-simple if and only if $\pi_{1}$ is a finite Abelian group (see Claim 3.13 for a precise statement).

The proof of the converse to (ii) is done in Section 2. It relies on a consequence of the proof of the main theorem in [3], which is formulated in Theorem 2.3. It is a replacement for the finite generation of the topological fundamental group in complex geometry (see the analogous statement in Remark 3.12). The proof of the converse to (iii) is done in Section 3. The key point to show the converse to (iii) is Theorem 3.4. Its proof relies on the construction (Proposition 3.5) of a moduli space of non-trivial extensions, with a rational map $f$ induced by Frobenius pullback, which allows us to use a theorem of Hrushovski.

ACKNOWLEDGEMENTS. We thank the department of Mathematics of Harvard University for its hospitality during the preparation of this note. We thank Nguyê̂n Duy Tân for a very careful reading of a first version, which allowed us to improve the redaction. We thank the referee of the first version who pointed out a mistake, corrected in Section 3. The second named author would like to thank his colleague Nanhua Xi for discussions related to Claim 3.3.

\section{Stratified bundles}

Let $k$ be an algebraically closed field of characteristic $p>0$, and $X$ be a smooth connected projective variety over $k$. A stratified bundle on $X$ is by definition a coherent $\mathcal{O}_{X}$-module $\mathcal{E}$ with a homomorphism

$$
\nabla: \mathcal{D}_{X} \rightarrow \operatorname{End}_{k}(\mathcal{E})
$$

of $\mathcal{O}_{X}$-algebras, where $\mathcal{D}_{X}$ is the sheaf of differential operators acting on the structure sheaf of $X$. By a theorem of Katz ( $c f$. [4, Theorem 1.3]), this is equivalent to 
the following definition. (The terminology is not unique: our stratified bundles are called flat bundles in [4], and F-divided sheaves in [2]).

Definition 2.1. A stratified bundle on $X$ is a sequence of bundles

$$
E=\left\{E_{0}, E_{1}, E_{2}, \cdots, \sigma_{0}, \sigma_{1}, \ldots\right\}=\left\{E_{i}, \sigma_{i}\right\}_{i \in \mathbb{N}}
$$

where $\sigma_{i}: F_{X}^{*} E_{i+1} \rightarrow E_{i}$ is an $\mathcal{O}_{X}$-linear isomorphism, and $F_{X}: X \rightarrow X$ is the absolute Frobenius.

A morphism $\alpha=\left\{\alpha_{i}\right\}:\left\{E_{i}, \sigma_{i}\right\} \rightarrow\left\{F_{i}, \tau_{i}\right\}$ between two stratified bundles is a sequence of morphisms $\alpha_{i}: E_{i} \rightarrow F_{i}$ of $\mathcal{O}_{X}$-modules such that

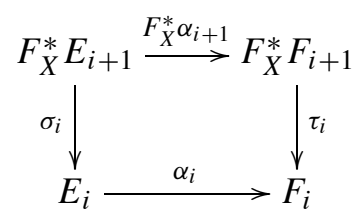

is commutative. The category $\operatorname{str}(X)$ of stratified bundles is Abelian, rigid, and monoidal. To see it is $k$-linear, it is better to define stratified bundles and morphisms in the relative version: objects consist of

$$
E^{\prime}=\left\{E_{0}=E_{0}^{\prime}, E_{1}^{\prime}, E_{2}^{\prime}, \cdots, \sigma_{0}^{\prime}, \sigma_{1}^{\prime}, \ldots\right\}=\left\{E_{i}^{\prime}, \sigma_{i}^{\prime}\right\}_{i \in \mathbb{N}}
$$

where $E_{i}^{\prime}$ is a bundle on the $i$-th Frobenius twist $X^{(i)}$ of $X$,

$$
\sigma_{i}^{\prime}: F_{i, i+1}^{*} E_{i+1}^{\prime} \rightarrow E_{i}^{\prime}
$$

is an $\mathcal{O}_{X^{(i)}}$-linear isomorphism, and $F_{i, i+1}: X^{(i)} \rightarrow X^{(i+1)}$ is the relative Frobenius, the morphisms are the obvious ones. A rational point $a \in X(k)$ yields a fiber functor $\omega_{a}: \operatorname{str}(X) \rightarrow \operatorname{vec}_{k}, \quad E \mapsto a^{*} E_{0}$ with values in the category of finite-dimensional vector spaces. Thus $\left(\operatorname{str}(X), \omega_{a}\right)$ is a Tannaka category $([2$, Section 2.2]), and one has an equivalence of categories

$$
\operatorname{str}(X) \stackrel{\omega_{a} \cong}{\longrightarrow} \operatorname{rep}_{k}\left(\pi^{\mathrm{str}}\right)
$$

where

$$
\pi^{\mathrm{str}}=\operatorname{Aut}^{\otimes}\left(\omega_{a}\right)
$$

is the Tannaka group scheme, and $\operatorname{rep}_{k}\left(\pi^{\mathrm{str}}\right)$ is the category of finite dimensional $k$-representations of $\pi^{\text {str }}$. Let $\pi_{1}:=\pi_{1}^{\text {ét }}(X, a)$ be the étale fundamental group of $X$. In [4, Theorem 1.10], D. Gieseker proved the following:

Theorem 2.2 (Gieseker). Let $X$ be a smooth projective variety over an algebraically closed field $k$. Then:

(i) If every stratified bundle is trivial, then $\pi_{1}$ is trivial;

(ii) If all the irreducible stratified bundles have rank 1 , then $\left[\pi_{1}, \pi_{1}\right]$ is a pro-pgroup; 
(iii) If every stratified bundle is a direct sum of stratified line bundles, then $\pi_{1}$ is Abelian with no p-power order quotient.

Then Gieseker conjectured that the converse of the above statements might be true. In [3], it is proven that the converse of statement (i) is true. The aim of this section is to prove the converse of (ii). We also prove the converse of (iii) in Section 3. The proof relies on the following theorem extracted from [3], and which plays a similar rôle as the finite generation of the topological fundamental group $\pi_{1}^{\text {top }}$ in complex geometry (see Remark 3.12). Fixing an ample line bundle $\mathcal{O}_{X}(1)$ on $X$, recall that $E$ is said to be $\mu$-stable if for all coherent subsheaves $U \subset E$ one has $\mu(U)<\mu(E)$, where $\mu(E)$ is the slope which is defined to be the degree of $E$ with respect to $\mathcal{O}_{X}(1)$ divided by the rank of $E$.

Theorem 2.3. Let $X$ be a smooth projective variety over an algebraically closed field $k$. If there is a stratified bundle $E=\left(E_{n}, \sigma_{n}\right)_{n \in \mathbb{N}}$ of rank $r \geq 2$ on $X$, where $\left\{E_{n}\right\}_{n \in \mathbb{N}}$ are $\mu$-stable, then there exists an irreducible representation $\rho: \pi_{1} \rightarrow$ $G L(V)$ with finite monodromy, where $V$ is an $r$-dimensional vector space over $\overline{\mathbb{F}} p$.

Proof. By the proof of [3, Theorem 3.15], there is a smooth projective model $X_{S} \rightarrow$ $S$ of $X \rightarrow$ Spec $k$, where $S$ is smooth affine over $\mathcal{F}_{p}$, with the following properties. The point $a \in X(k)$ lifts to $a_{S} \in X_{S}(S)$. There is a quasi-projective model $M_{S} \rightarrow S$ of the moduli of stable vector bundles considered in [3, Section 3]. There is a closed point $u \rightarrow M_{S}$ of residue field $\mathbb{F}_{q}$, corresponding to a bundle $\mathcal{E}$ over $X_{S}$, stable over $X_{\bar{s}}=X \otimes \overline{\mathbb{F}}_{p}$, where $s$ is the closed point $u$ viewed as a closed point of $S$, such that $\left(F^{m}\right)^{*} \mathcal{E} \cong \mathcal{E}$, for some $m \in \mathbb{N} \backslash\{0\}$, where $F$ is the Frobenius of $X_{s} / \mathbb{F}_{q}$.

Thus, as explained in [3, Theorem 3.15], $\mathcal{E}$ trivializes over a Lang torsor $Y \rightarrow X_{s}\left[9\right.$, Satz 1.4], thus its base change $\mathcal{E}_{\bar{s}}$ to $X_{\bar{s}}$ trivializes over $Y \times{ }_{s} \bar{s} \rightarrow X_{\bar{s}}$. This trivialization defines an irreducible representation $\rho^{\prime}: \pi_{1}^{\text {ét }}\left(X_{\bar{s}}, b\right) \rightarrow G L\left(\mathcal{E}_{b}\right)$, where $b=a_{S} \otimes \bar{s}$. As the specialization homomorphism sp : $\pi_{1} \rightarrow \pi_{1}^{\text {ét }}\left(X_{\bar{s}}, b\right)$ is surjective ([13, Exposé X, Théorème 3.8]), the composite $\rho=\mathrm{sp} \circ \rho^{\prime}$ is a representation of $\pi_{1}$ with the same finite irreducible monodromy. This completes the proof.

We now use the following two elementary lemmas.

Lemma 2.4 (See [12, Chapter 8, Proposition 26]). Let $G$ be a finite p-group and $\rho: G \rightarrow G L(V)$ be a representation on a vector space $V \neq 0$ over a field $k$ of characteristic $p$. Then

$$
V^{G}=\{v \in V \mid \rho(g) v=v \forall g \in G\} \neq 0 .
$$

Lemma 2.5. Let $G$ be a finite group and $\rho: G \rightarrow G L(V)$ be an irreducible representation on a finite dimensional vector space $V$ over an algebraically closed field $k$ of characteristic $p>0$. If the commutator $[G, G]$ of $G$ is a p-group, then $\operatorname{dim}(V)=1$. 
Proof. If $[G, G]$ is a $p$-group, by Lemma 2.4 , there exists a $0 \neq v \in V$ such that $\rho\left(g_{1}\right) \rho\left(g_{2}\right) v=\rho\left(g_{2}\right) \rho\left(g_{1}\right) v$ for any $g_{1}, g_{2} \in G$. Since $V$ is an irreducible $G$ module, the sub-vector space spanned by the orbit $\rho(G) v$ is $V$ itself. Thus for all $w \in V$, all $g \in G$, there are $a_{g}(w) \in k$, such that

$$
w=\sum_{g \in G} a_{g}(w) \rho(g) v .
$$

On the other hand, for all $g_{1}, g_{2}, g \in G$, one has $g_{1} g_{2}=g_{2} g_{1} c$ for some $c \in$ $[G, G]$, thus $g_{1} g_{2} g=g_{2} g_{1} c g=g_{2} g_{1} g c^{\prime}$, for some $c^{\prime} \in[G, G]$. We conclude that $\rho\left(g_{1}\right) \rho\left(g_{2}\right) w=\rho\left(g_{2}\right) \rho\left(g_{1}\right) w$ for any $g_{1}, g_{2} \in G$, that is $\rho(G)$ is Abelian. As $\rho$ is irreducible, $V$ must have dimension 1 .

Theorem 2.6. Let $X$ be a smooth projective variety over an algebraically closed field $k$. Then all irreducible stratified bundles on $X$ have rank 1 if and only if the commutator $\left[\pi_{1}, \pi_{1}\right]$ of $\pi_{1}$ is a pro-p-group.

Proof. One direction is the (ii) in Theorem 2.2. We prove the converse. Assume that $\left[\pi_{1}, \pi_{1}\right]$ is a pro- $p$-group. Let $E=\left(E_{n}, \sigma_{n}\right)_{n \in \mathbb{N}}$ be an irreducible stratified bundle of rank $r \geq 1$ on $X$. Assume $r \geq 2$. Then by [3, Proposition 2.3], there is an $n_{0} \in \mathbb{N}$ such that the stratified bundle $E\left(n_{0}\right):=\left(E_{n}, \sigma_{n}\right)_{n \geq n_{0}}$ is a successive extension of stratified bundles $U=\left(U_{n}, \tau_{n}\right)$ with underlying bundles $U_{n}$ being $\mu$-stable. But $E$ being irreducible implies that $E\left(n_{0}\right)$ is irreducible as well. Hence all the $E_{n}$ are $\mu$-stable for $n \geq n_{0}$. By Theorem 2.3, there is an irreducible representation $\rho: \pi_{1} \rightarrow G L(V)$ of dimension $r \geq 2$ with finite monodromy over an algebraically closed field of characteristic $p>0$. By Lemma 2.5, this is impossible. Thus $r=1$.

\section{Extensions of stratified line bundles}

In this section we prove the following:

Theorem 3.1. Let X be a smooth projective connected variety over an algebraically closed field $k$ of characteristic $p>0$. If the étale fundamental group $\pi_{1}$ of $X$ is Abelian and has no non-trivial p-power order quotient, then any extension

$$
0 \rightarrow \mathbb{L} \rightarrow \mathbb{V} \rightarrow \mathbb{L}^{\prime} \rightarrow 0
$$

in $\operatorname{str}(\mathrm{X})$ is split when $\mathbb{L}$ and $\mathbb{L}^{\prime}$ are rank-1 objects.

We start the proof by the following result, which can be considered as a generalization of [3, Proposition 2.4]:

Proposition 3.2. Let $L$ be a line bundle on $X$ such that $\left(F_{X}^{*}\right)^{a} L$ is isomorphic to $L$, for some non zero natural number $a$, where $F_{X}: X \rightarrow X$ is the absolute Frobenius map. Then

$$
\left(F_{X}^{*}\right)^{a}: H^{1}(X, L) \rightarrow H^{1}(X, L)
$$

is nilpotent if $\pi_{1}$ is Abelian without non-trivial p-power quotient. 
Proof. Since $\left(F_{X}^{*}\right)^{a} L$ is isomorphic to $L$, we have that $L$ is a torsion line bundle of order $n$ prime to $p$. The choice of an isomorphism $L^{n} \cong \mathcal{O}_{X}$ defines a Kummer cover $\phi: Y \rightarrow X$ with Galois group $H=\mathbb{Z} / n$ such that

$$
\phi^{*} L \cong \mathcal{O}_{Y}
$$

One has an exact sequence (recall that $\pi_{1}:=\pi_{1}^{\text {ét }}(X, \bar{a})$ )

$$
1 \rightarrow \pi_{1}^{\mathrm{e} t}(Y, \bar{b}) \rightarrow \pi_{1} \rightarrow H \rightarrow 1,
$$

where $\bar{b}$ is a geometric point of $Y$ above the geometric point $\bar{a}$ of $X$.

Claim 3.3. $\pi_{1}^{\text {ét }}(Y, \bar{b})$ is Abelian without $p$-power quotient.

Proof. Since $\pi_{1}$ is commutative, the kernel of any quotient map

$$
\pi_{1}^{\mathrm{e} t}(Y, \bar{b}) \rightarrow K
$$

is normal in $\pi_{1}$. Taking for $K$ a finite $p$-group defines the push-out exact sequence

$$
1 \rightarrow K \rightarrow G \rightarrow H \rightarrow 1
$$

where $G$ is a quotient of $\pi_{1}$, thus is commutative and has no $p$-power quotient. Since $K$ splits in $G, K=\{1\}$.

Then, by [3, Proposition 2.4], there is an integer $N>0$ such that

$$
\left(F_{Y}^{*}\right)^{N}: H^{1}\left(Y, \mathcal{O}_{Y}\right) \rightarrow H^{1}\left(Y, \mathcal{O}_{Y}\right)
$$

is a zero map, which implies that

$$
\phi^{*} \cdot\left(F_{X}^{*}\right)^{N a}=\left(F_{Y}^{*}\right)^{N a} \cdot \phi^{*}: H^{1}(X, L) \rightarrow H^{1}\left(Y, \mathcal{O}_{Y}\right)
$$

is a zero map. But $\phi^{*}: H^{1}(X, L) \hookrightarrow H^{1}\left(Y, \phi^{*} L\right)=H^{1}\left(Y, \mathcal{O}_{Y}\right)$ is injective (since $\mathcal{O}_{X} \hookrightarrow \phi_{*} \mathcal{O}_{Y} \rightarrow \oplus_{i} L^{-i}$ is split by the construction of $\phi: Y \rightarrow X$ ), thus $\left(F_{X}^{*}\right)^{N a}: H^{1}(X, L) \rightarrow H^{1}(X, L)$ is a zero map.

Proof of Theorem 3.1. By twisting with $\left(\mathbb{L}^{\prime}\right)^{-1}$, we may assume that $\mathbb{L}^{\prime}=\mathbb{I}$, the trivial object in $\operatorname{str}(X)$. We prove Theorem 3.1 by contradiction. If there exists a nontrivial extension

$$
0 \rightarrow \mathbb{L} \rightarrow \mathbb{V} \rightarrow \mathbb{I} \rightarrow 0
$$

in $\operatorname{str}(X)$, then, by definition, there is a set

$$
\mathbb{E}_{X}=\left\{\left(L_{i} \hookrightarrow V_{i} \rightarrow \mathcal{O}_{X}\right)\right\}_{i \in \mathbb{N}}
$$


of isomorphism classes of non-trivial extensions on $X$ such that

$$
\left(L_{i} \hookrightarrow V_{i} \rightarrow \mathcal{O}_{X}\right) \cong\left(F_{X}^{*} L_{i+1} \hookrightarrow F_{X}^{*} V_{i+1} \rightarrow F_{X}^{*} \mathcal{O}_{X}\right)
$$

for any $i \in \mathbb{N}$. In particular, $L_{i}$ is isomorphic as a line bundle to $F_{X}^{*}\left(L_{i+1}\right)$. We will consider the moduli point in $\mathbb{P}\left(H^{1}\left(L_{i}\right)\right)$ of a nontrivial extension $\left(L_{i} \hookrightarrow V_{i} \rightarrow\right.$ $\left.\mathcal{O}_{X}\right) \in H^{1}\left(L_{i}\right)$. Thus, when we say that $\mathbb{E}_{X}$ is a finite (respectively, infinite) set, it means that the set of isomorphism classes of extensions is a finite (respectively, infinite) set modulo scale.

If $\mathbb{E}_{X}$ is a finite set, then there is a non-trivial extension

$$
\left(L_{i} \hookrightarrow V_{i} \rightarrow \mathcal{O}_{X}\right) \in \mathbb{E}_{X}
$$

and an integer $a>0$ such that

$$
\left(F_{X}^{*}\right)^{a}\left(L_{i} \hookrightarrow V_{i} \rightarrow \mathcal{O}_{X}\right) \cong\left(L_{i} \hookrightarrow V_{i} \rightarrow \mathcal{O}_{X}\right) .
$$

In particular, $\left(F_{X}^{*}\right)^{a} L_{i} \cong L_{i}$. By Proposition 3.2,

$$
\left(F_{X}^{*}\right)^{a}: H^{1}\left(X, L_{i}\right) \rightarrow H^{1}\left(X, L_{i}\right)
$$

is nilpotent. We obtain a contradiction since $\left(F_{X}^{*}\right)^{a}$ sends the extension class $e_{i}=$ $\left(L_{i} \hookrightarrow V_{i} \rightarrow \mathcal{O}_{X}\right) \in \mathbb{P}\left(H^{1}\left(X, L_{i}\right)\right)$ to itself.

If $\mathbb{E}_{X}$ is a infinite set, by Theorem 3.4 below, there is a nontrivial extension

$$
0 \rightarrow L \rightarrow V \rightarrow \mathcal{O}_{X_{\bar{s}}} \rightarrow 0
$$

on a good reduction $X_{\bar{s}}$ of $X$ (thus over $\overline{\mathbb{F}}_{p}$ ) such that for an integer $a>0$

$$
0 \rightarrow\left(F_{X_{\bar{s}}}^{*}\right)^{a} L \rightarrow\left(F_{X_{\bar{s}}}^{*}\right)^{a} V \rightarrow\left(F_{X_{\bar{S}}}^{*}\right)^{a} \mathcal{O}_{X_{\bar{s}}} \rightarrow 0
$$

is isomorphic to the extension (3.2). Then, by using Proposition 3.2 to $X_{\bar{s}}$, we obtain again a contradiction since $\pi_{1}^{\text {ét }}\left(X_{\bar{s}}, a_{\bar{s}}\right)$, which is a quotient of $\pi_{1}$ via the specialization map, is also Abelian without non-trivial $p$-power quotient. Here $a_{\bar{s}}$ is a specialization of the geometric point $\bar{a}$ used to define $\pi_{1}$.

Theorem 3.4. Let $X$ be a smooth projective variety over an algebraically closed field $k$. If there exists an infinite set $\mathbb{E}_{X}$ of equivalence classes of nontrivial extensions

$$
\left(L_{i} \hookrightarrow V_{i} \rightarrow \mathcal{O}_{X}\right), \quad i \in \mathbb{N}
$$

satisfying $\left(L_{i} \hookrightarrow V_{i} \rightarrow \mathcal{O}_{X}\right) \cong\left(F_{X}^{*} L_{i+1} \hookrightarrow F_{X}^{*} V_{i+1} \rightarrow F_{X}^{*} \mathcal{O}_{X}\right)$ for any $i \in \mathbb{N}$, then there exists a nontrivial extension

$$
0 \rightarrow L \rightarrow V \rightarrow \mathcal{O}_{X_{\bar{s}}} \rightarrow 0
$$

on a good reduction $X_{\bar{s}}$ of $X\left(\right.$ over $\left.\overline{\mathbb{F}}_{p}\right)$ such that for some a $>0$

$$
\left(\left(F_{X_{\bar{s}}}^{*}\right)^{a} L \hookrightarrow\left(F_{X_{\bar{s}}}^{*}\right)^{a} V \rightarrow\left(F_{X_{\bar{s}}}^{*}\right)^{a} \mathcal{O}_{X_{\bar{s}}}\right) \cong\left(L \hookrightarrow V \rightarrow \mathcal{O}_{X_{\bar{s}}}\right) .
$$


We now prove Theorem 3.4. Let $X_{S} \rightarrow S$ be a smooth projective model of $X / k$, with geometrically irreducible fibers, endowed with a section, where $S$ is a smooth affine irreducible variety over $\mathbb{F}_{q}$. Then we have:

Proposition 3.5. There exists a reduced $S$-scheme $M \rightarrow S$ of finite type and a rational map $f: M \rightarrow M$ over $S$ such that:

(1) For any field extension $K \supset \mathbb{F}_{q}$, the set $M(K)$ of $K$-valued points consists of isomorphism classes of non-trivial extensions $e: L \hookrightarrow V \rightarrow \mathcal{O}_{X_{s}}$ on $X_{S}$, where $X_{s}$ is the fiber of $X_{S} \rightarrow S$ at the image $s \in S(K)$ of $e \in M(K)$;

(2) The set $\mathbb{E}_{X}$ of Theorem 3.4 minus finitely many elements lies in $M(k)$;

(3) The rational map $f: M \rightarrow M$ is well-defined at $e=\left(L \hookrightarrow V \rightarrow \mathcal{O}_{X_{s_{0}}}\right) \in$ $M(K)$ if and only if the pull-back extension

$$
0 \rightarrow F_{X_{s}}^{*} L \rightarrow F_{X_{s}}^{*} V \rightarrow F_{X_{s}}^{*} \mathcal{O}_{X_{s}} \rightarrow 0
$$

under the absolute Frobenius $F_{X_{s}}$ does not split, in which case

$$
f(e)=\left(F_{X_{s}}^{*} L \hookrightarrow F_{X_{s}}^{*} V \rightarrow F_{X_{s}}^{*} \mathcal{O}_{X_{s}}\right) \in M(K) .
$$

Proof. The line bundles $\left\{L_{i}\right\}_{i \in \mathbb{N}}$ occurring in $\mathbb{E}_{X}$ must satisfy

$$
L_{i} \cong L_{i+1}^{p}, \quad \forall i \in \mathbb{N},
$$

so they are infinitely $p$-divisible, thus lie in $\operatorname{Pic}^{\tau}(X)(k)$, the group of numerically trivial line bundles over $k([8,9.6])$.

We first assume $\mathbb{L}=\mathbb{I}$. We shrink $S$ so that $H^{1}\left(\mathcal{O}_{X_{S}}\right)$ is locally free and commutes with base change. Let $H^{1}\left(\mathcal{O}_{X_{S}}\right)$ denote the first direct image of $\mathcal{O}_{X_{S}}$ under $X_{S} \rightarrow S$, then

$$
\pi_{S}: M=\mathbb{P}\left(H^{1}\left(\mathcal{O}_{X_{S}}\right)^{\vee}\right) \rightarrow S
$$

is the moduli space of isomorphism classes of non-trivial extensions $\mathcal{O}_{X_{\bar{s}}} \hookrightarrow V \rightarrow$ $\mathcal{O}_{X_{\bar{s}}}$. In the diagram

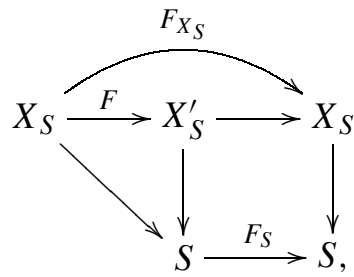

$F_{S}, F_{X_{S}}$ are the absolute Frobenius and $F: X_{S} \rightarrow X_{S}^{\prime}$ is the relative Frobenius. Then the "Frobenius pullback" induces

$$
F^{*}: F_{S}^{*} H^{1}\left(\mathcal{O}_{X_{S}}\right)=H^{1}\left(\mathcal{O}_{X_{S}^{\prime}}\right) \rightarrow H^{1}\left(\mathcal{O}_{X_{S}}\right)
$$

which is $\mathcal{O}_{S}$-linear and commutes with base change. Let

$$
\pi_{S}^{*} H^{1}\left(\mathcal{O}_{X_{S}}\right)^{\vee} \rightarrow \mathcal{O}_{M}(1) \rightarrow 0
$$


be the universal rank-one quotient, which, pulled back by the absolute Frobenius $F_{M}: M \rightarrow M$, defines the $\mathcal{O}_{M}$-linear map

$$
\pi_{S}^{*} F_{S}^{*} H^{1}\left(\mathcal{O}_{X_{S}}\right)^{\vee} \rightarrow \mathcal{O}_{M}(p) \rightarrow 0
$$

(since $F_{M}^{*} \pi_{S}^{*}=\pi_{S}^{*} F_{S}^{*}$ and $F_{M}^{*} \mathcal{O}_{M}(1):=\mathcal{O}_{M}(p)$ ). Combining the dual

$$
\pi_{S}^{*}\left(F^{* \vee}\right): \pi_{S}^{*} H^{1}\left(\mathcal{O}_{X_{S}}\right)^{\vee} \rightarrow \pi_{S}^{*} F_{S}^{*} H^{1}\left(\mathcal{O}_{X_{S}}\right)^{\vee}
$$

of (3.3) with (3.4) defines the $\mathcal{O}_{M}$-linear map

$$
\pi_{S}^{*} H^{1}\left(\mathcal{O}_{X_{S}}\right)^{\vee} \rightarrow \mathcal{O}_{M}(p) .
$$

By the universal property, this is the same as a rational map

$$
f: M \rightarrow M
$$

over $S$. To see that $f$ satisfies (3), let $e \rightarrow M$ be a point with image $s \rightarrow S$ (under $M \stackrel{\pi_{S}}{\longrightarrow} S$ ), which corresponds to a nontrivial extension

$$
e=\left(\mathcal{O}_{X_{s}} \hookrightarrow V \rightarrow \mathcal{O}_{X_{s}}\right)
$$

where $X_{s}$ is the fiber of $X_{S} \rightarrow S$ at $s \rightarrow S$. Then $f$ is well-defined at $e \rightarrow M$ if and only if the $\mathcal{O}_{M}$-linear map

$$
\pi_{S}^{*} H^{1}\left(\mathcal{O}_{X_{S}}\right)^{\vee} \rightarrow \mathcal{O}_{M}(p)
$$

at $e \rightarrow M$ is surjective. By definition, this is equivalent to saying that the $\mathcal{O}_{S}$-linear map (3.3) at $s \rightarrow S$, which is

$$
F^{*}: F_{k(s)}^{*} H^{1}\left(\mathcal{O}_{X_{s}}\right)=H^{1}\left(\mathcal{O}_{X_{s}^{\prime}}\right) \rightarrow H^{1}\left(\mathcal{O}_{X_{s}}\right),
$$

is not trivial at $F_{k(s)}^{*}([e]) \in H^{1}\left(\mathcal{O}_{X_{s}^{\prime}}\right)\left(\right.$ i.e. $\left.F^{*}\left(F_{k(s)}^{*}([e])\right) \neq 0\right)$, where

$$
[e] \in H^{1}\left(\mathcal{O}_{X_{S}}\right)
$$

denotes class corresponding to $e=\left(\mathcal{O}_{X_{s}} \hookrightarrow V \rightarrow \mathcal{O}_{X_{S}}\right)$. Since

$$
F_{X_{s}}^{*}: H^{1}\left(\mathcal{O}_{X_{s}}\right) \stackrel{F_{k(s)}^{*}}{\longrightarrow} H^{1}\left(\mathcal{O}_{X_{s}^{\prime}}\right) \stackrel{F^{*}}{\longrightarrow} H^{1}\left(\mathcal{O}_{X_{s}}\right),
$$

we have $F_{X_{s}}^{*}([e])=F^{*}\left(F_{k(s)}^{*}([e])\right)$. Thus $f$ is well-defined at

$$
e=\left(\mathcal{O}_{X_{s}} \hookrightarrow V \rightarrow \mathcal{O}_{X_{s}}\right) \in M
$$

if and only if $\left(F_{X_{s}}^{*} \mathcal{O}_{X_{S}} \hookrightarrow F_{X_{S}}^{*} V \rightarrow F_{X_{S}}^{*} \mathcal{O}_{X_{S}}\right)$ is not splitting, and

$$
f(e)=\left(F_{X_{S}}^{*} \mathcal{O}_{X_{S}} \hookrightarrow F_{X_{s}}^{*} V \rightarrow F_{X_{S}}^{*} \mathcal{O}_{X_{s}}\right) .
$$


The objects $\left(\mathcal{O}_{X} \hookrightarrow V_{i} \rightarrow \mathcal{O}_{X}\right) \in \mathbb{E}_{X}(i \in \mathbb{N})$ define points $e_{i} \rightarrow M$ over $s=\operatorname{Spec}(k) \rightarrow S$, and $f$ is well-defined at $e_{i}$ with $f\left(e_{i}\right)=e_{i-1}$ for $i>1$. This finishes the proof if $\mathbb{L}=\mathbb{I}$.

If $\mathbb{L} \neq \mathbb{I}$, after removing a finite number of elements in the set $\mathbb{E}_{X}$, we can assume that all line bundles $\left\{L_{i}\right\}_{i \in \mathbb{N}}$ occurring in $\mathbb{E}_{X}$ satisfy

$$
H^{0}\left(X, L_{i}\right)=0, H^{1}\left(L_{i}\right) \neq 0 \quad i \in \mathbb{N}
$$

( $L_{i} \neq \mathcal{O}_{X}$ if and only if $H^{0}\left(L_{i}\right) \neq 0$ since $L_{i}$ is numerically trivial). Let $\mathrm{Pic}_{X_{S}}^{\tau} \rightarrow$ $S$ be the torsion component of the identity of the Picard scheme $[8,9.6]$. Let $\mathcal{L}$ be the universal line bundle on $X_{S} \times{ }_{S} \operatorname{Pic}_{X_{S}}^{\tau}$. We define

$$
\mathcal{N}_{0}=\left\{t \in \operatorname{Pic}_{X_{S}}^{\tau} \mid H^{1}\left(\mathcal{L}_{t}\right) \neq 0\right\}
$$

with its reduced structure. By the semi-continuity of cohomology, this is a closed sub-scheme of $\operatorname{Pic}_{X_{S}}^{\tau}$. If $\mathcal{N}_{i}$ is defined, let

$$
\mathcal{N}_{i+1}=\left\{t \in \mathcal{N}_{i} \mid H^{1}\left(\mathcal{L}_{t}^{p^{i+1}}\right) \neq 0\right\}
$$

By the Nötherian property, $\mathcal{N}_{0} \supseteq \mathcal{N}_{1} \supseteq \ldots \mathcal{N}_{i} \supseteq \mathcal{N}_{i+1} \supseteq \cdots$ terminates. Then, there is a $k_{0} \geq 0$ such that

$$
\mathcal{N}_{i}=\mathcal{N}_{k_{0}} \quad \forall i \geq k_{0}
$$

The line bundles $\left\{L_{i+p^{k^{0}}}\right\}_{i \in \mathbb{N}}$ occurring in $\mathbb{E}_{X}\left(p^{k_{0}}\right)$, where

$$
\mathbb{E}_{X}\left(p^{k_{0}}\right):=\left\{\left(L_{i+p^{k_{0}}} \hookrightarrow V_{i+p^{k_{0}}} \rightarrow \mathcal{O}_{X}\right) \in \mathbb{E}_{X}\right\}_{i \in \mathbb{N}},
$$

are $k$-points of $\mathcal{N}_{k_{0}}=\mathcal{N}_{i}$ for all $i \geq k_{0}$.

We define $T \subset \mathcal{N}_{k_{0}} \rightarrow S$ with its reduced structure to be the sub-scheme

$$
T=\left\{t \in \mathcal{N}_{k_{0}} \mid H^{0}\left(\mathcal{L}_{t}\right)=0\right\}
$$

Such a $T$ is open in $\mathcal{N}_{k_{0}}$. Let $\mathcal{L}$ be the restriction of universal line bundle on $X_{S} \times{ }_{S} T$ (thus, for any $t \in T, H^{1}\left(\mathcal{L}_{t}^{p^{i}}\right) \neq 0$ for $i \geq 0$ ). Unfortunately, $R^{1} p_{T *}(\mathcal{L})$ is neither commuting with base change nor locally free in general, where $p_{T}: X_{S} \times{ }_{S} T \rightarrow T$ is the projection. However,

$$
\mathcal{E}=R^{n-1} p_{T *}\left(\mathcal{L}^{\vee} \otimes p_{X_{S}}^{*} \omega_{X_{S} / S}\right), \quad n=\operatorname{dim}(X)
$$

may not be locally free, but does commute with base change since

$$
H^{n}\left(\mathcal{L}_{t}^{\vee} \otimes \omega_{X_{S} \times\{t\}}\right)=H^{0}\left(\mathcal{L}_{t}\right)^{\vee}=0 \quad \forall t \in T
$$


There exists a quotient scheme $\pi: M=\mathbb{P}(\mathcal{E}) \rightarrow T$ together with

$$
\pi^{*} \mathcal{E} \rightarrow \mathcal{O}_{M}(1) \rightarrow 0,
$$

which represents the functor that sends a $T$-scheme $p_{W}: W \rightarrow T$ to the set of isomorphic classes of quotients $p_{W}^{*} \mathcal{E} \rightarrow Q \rightarrow 0$, where $Q$ is a line bundle on $W$ ([7, Theorem 2.2.4]). By definition, $M \stackrel{\pi}{\rightarrow} T \rightarrow S$ satisfies the properties (1) and (2) in the proposition (replacing $M$ by $M_{\text {red }}$ if necessary). The rest of the proof is devoted to the construction of a rational map

$$
f: M \rightarrow M
$$

over $S$, which satisfies the property (3) in the proposition.

Let $F: X_{S} \times{ }_{S} T \rightarrow\left(X_{S} \times{ }_{S} T\right)^{\prime}$ denote the relative Frobenius morphism over $T$. We write

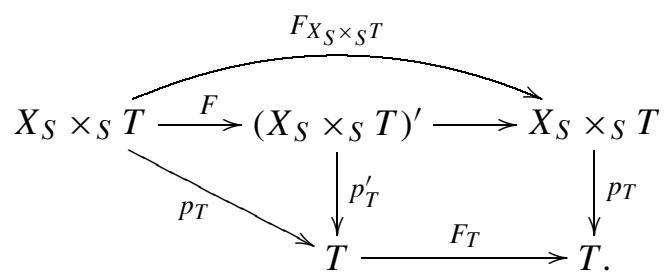

One has $\left(X_{S} \times{ }_{S} T\right)^{\prime}=X_{S}^{\prime} \times_{S} T$ thus we can project to $X_{S}^{\prime}$, further to $X_{S}$. Let $\mathcal{L}^{\prime}$ be the pullback of $\mathcal{L}$ under the projection

$$
\left(X_{S} \times S T\right)^{\prime} \stackrel{p_{1}}{\rightarrow} X_{S} \times{ }_{S} T
$$

and $\omega$ (respectively $\omega^{\prime}$ ) be the relative dualizing sheaf of $X_{S} \times{ }_{S} T \stackrel{p_{T}}{\longrightarrow} T$ (respectively $\left.\left(X_{S} \times{ }_{S} T\right)^{\prime} \stackrel{p_{T}^{\prime}}{\longrightarrow} T\right)$. The line bundle $F^{*} \mathcal{L}^{\prime}$ on $X_{S} \times{ }_{S} T$ defines a $S$-morphism $v: T \rightarrow \operatorname{Pic}_{X_{S}}^{\tau}$. Define

$$
T^{0}=\left\{t \in T \mid H^{0}\left(\mathcal{L}_{t}^{p}\right)=0\right\} \subset T
$$

with its reduced scheme structure. Note that $T^{0}$ is open in $T$. By definition of $T, v$ restricted to $T^{0}$ factors through $T$, thus it induces

$$
v: T^{0} \rightarrow T
$$

such that

$$
(1 \times \nu)^{*} \mathcal{L} \otimes p_{T^{0}}^{*} \eta=F^{*} \mathcal{L}^{\prime}
$$

on $X_{S} \times{ }_{S} T^{0}$, with $X_{S} \times{ }_{S} T^{0} \stackrel{p_{T^{0}}}{\longrightarrow} T^{0}$, and where $\eta$ is a line bundle on $T^{0}$. We abuse notation and still write $\omega$ for the relative dualizing sheaf $\omega_{X_{S} \times{ }_{S} T^{0} / T^{0}}$ of $p_{T^{0}}$, 
$\omega^{\prime}$ for the relative dualizing sheaf $\omega_{X_{S}^{\prime} \times{ }_{S} T^{0} / T^{0}}$ of $p_{T^{0}}^{\prime}: X_{S}^{\prime} \times{ }_{S} T^{0} \rightarrow T^{0}$. Since $\omega=p_{X_{S}}^{*} \omega_{X_{S} / S}=(1 \times v)^{*} \omega_{X_{S} \times{ }_{S} T / T}$, we have

$$
R^{n-1} p_{T^{0} *}\left(\omega \otimes F^{*} \mathcal{L}^{\prime \vee}\right)=\eta^{-1} \otimes v^{*} \mathcal{E}, \quad R^{n-1} p_{T^{0} *}^{\prime}\left(\omega^{\prime} \otimes \mathcal{L}^{\prime \vee}\right)=F_{T^{0}}^{*} \mathcal{E},
$$

and these identities commute with base change. By Lemma 3.6, we have

$$
\phi: \eta^{-1} \otimes v^{*} \mathcal{E} \rightarrow F_{T^{0}}^{*} \mathcal{E}
$$

Together with the universal quotient $\pi^{*} \mathcal{E} \rightarrow \mathcal{O}_{M_{0}}(1) \rightarrow 0$, restricted to $M_{0}:=$ $M \times{ }_{S} T^{0}$, where $\pi: M_{0} \rightarrow T^{0}, T^{0} \rightarrow S$ factors through the composition of the open embedding $T^{0} \subset T$ with the map $T \rightarrow S$, this induces

$$
\Phi: \pi^{*} \nu^{*} \mathcal{E} \stackrel{\phi}{\rightarrow} \pi^{*} \eta \otimes \pi^{*} F_{T^{0}}^{*} \mathcal{E}=\pi^{*} \eta \otimes F_{M_{0}}^{*} \pi^{*} \mathcal{E} \rightarrow \pi^{*} \eta \otimes \mathcal{O}_{M_{0}}(p)
$$

Here $F_{T^{0}}, F_{M_{0}}$ are the absolute Frobenius morphisms satisfying

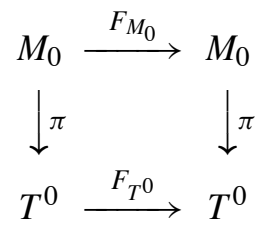

Let $M^{0} \subset M$ be the reduced open set consisting of points $q \in M$ such that $\Phi$ is surjective at $q \in M$ (which implies $M^{0} \subset M_{0}$ ). Then there exists a unique morphism

$$
f: M^{0} \rightarrow M
$$

corresponding via the universal property to $f^{*}\left(\pi^{*} \mathcal{E} \rightarrow \mathcal{O}_{M}(1) \rightarrow 0\right)=\left(\pi^{*} \nu^{*} \mathcal{E} \rightarrow\right.$ $\left.\pi^{*} \eta \otimes \mathcal{O}_{M^{0}}(p) \rightarrow 0\right)$. By definition, one has the factorization

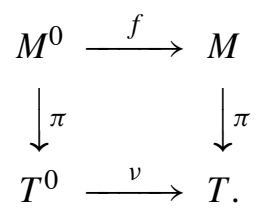

Then Lemma 3.6 below implies that the rational map

$$
f: M \rightarrow M
$$

satisfies the requirement (3) in the proposition. 
For any point $t \rightarrow T$, let $s \rightarrow S$ be its image under $T \rightarrow S$, and $X_{S}$ be the fiber of $X_{S} \rightarrow S$ at $s \rightarrow S$. Then the diagram (3.5) specializes to

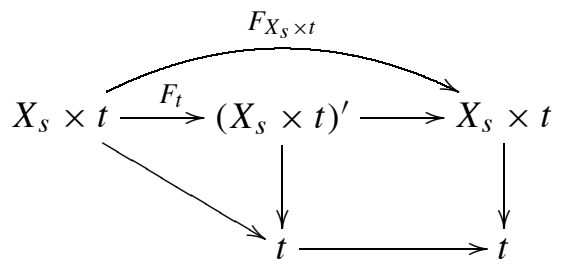

and $F_{t}$ induces the $k(t)$-linear map $F_{t}^{*}: H^{1}\left(\mathcal{L}_{t}^{\prime}\right) \rightarrow H^{1}\left(\left(F^{*} \mathcal{L}^{\prime}\right)_{t}\right)$, which induces a $k(t)$-linear map

$$
\left(F_{t}^{*}\right)^{\vee}: H^{1}\left(\left(F^{*} \mathcal{L}^{\prime}\right)_{t}\right)^{\vee} \rightarrow H^{1}\left(\mathcal{L}_{t}^{\prime}\right)^{\vee}
$$

By Serre duality, we have the induced $k(t)$-linear map

$$
\phi_{t}: H^{n-1}\left(\left(\omega \otimes F^{*} \mathcal{L}^{\prime \vee}\right)_{t}\right) \rightarrow H^{n-1}\left(\left(\omega^{\prime} \otimes \mathcal{L}^{\prime \vee}\right)_{t}\right) .
$$

Serre duality allows to make (3.6) in families:

Lemma 3.6. There exists a homomorphism

$$
\phi: R^{n-1} p_{T_{*}}\left(\omega \otimes F^{*} \mathcal{L}^{\prime \vee}\right) \rightarrow R^{n-1} p_{T *}^{\prime}\left(\omega^{\prime} \otimes \mathcal{L}^{\prime \vee}\right)
$$

such that

$$
\begin{array}{ccc}
R^{n-1} p_{T *}\left(\omega \otimes F^{*} \mathcal{L}^{\prime \vee}\right) \otimes k(t) & \stackrel{\tau}{\longrightarrow} H^{n-1}\left(\left(\omega \otimes F^{*} \mathcal{L}^{\prime \vee}\right)_{t}\right) \\
\downarrow \phi \otimes k(t) & & \downarrow_{t} \\
R^{n-1} p_{T *}^{\prime}\left(\omega^{\prime} \otimes \mathcal{L}^{\prime \vee}\right) \otimes k(t) & \stackrel{\tau}{\longrightarrow} & H^{n-1}\left(\left(\omega^{\prime} \otimes \mathcal{L}^{\prime \vee}\right)_{t}\right)
\end{array}
$$

is commutative for any $t \rightarrow T$, where $\tau$ is the canonical base change isomorphism, and $\phi_{t}$ is the homomorphism in (3.6).

Proof. Let $\omega$ (respectively, $\omega^{\prime}$ ) be the relative canonical line bundle of

$$
X_{T}:=X_{S} \times{ }_{S} T \stackrel{p_{T}}{\longrightarrow} T
$$

(respectively, $\left.X_{T}^{\prime}:=\left(X_{S} \times_{S} T\right)^{\prime} \stackrel{p_{T}^{\prime}}{\longrightarrow} T\right)$. Let $F: X_{T} \rightarrow X_{T}^{\prime}$ be the relative Frobenius morphism in (3.5), which is flat and Cohen-Macaulay as $X_{S} \rightarrow S$ is smooth. The injection $\mathcal{L}^{\prime} \hookrightarrow F_{*} F^{*} \mathcal{L}^{\prime}$ of vector bundles induces the surjection of vector bundles

$$
\operatorname{Hom}\left(F_{*} F^{*} \mathcal{L}^{\prime}, \omega^{\prime}\right) \rightarrow \operatorname{Hom}\left(\mathcal{L}^{\prime}, \omega^{\prime}\right) .
$$

Duality theory for the Cohen-Macaulay map $F: X_{T} \rightarrow X_{T}^{\prime}$ implies an isomorphism

$$
F_{*} \operatorname{Hom}\left(F^{*} \mathcal{L}^{\prime}, \omega\right) \cong \operatorname{Hom}\left(F_{*} F^{*} \mathcal{L}^{\prime}, \omega^{\prime}\right)
$$


Equations (3.7) and (3.8) imply that one has a surjection

$$
F_{*} \operatorname{Hom}\left(F^{*} \mathcal{L}^{\prime}, \omega\right) \rightarrow \operatorname{Hom}\left(\mathcal{L}^{\prime}, \omega^{\prime}\right)
$$

of vector bundles on $X_{S}^{\prime}$. Since $F$ is a finite morphism, taking $R^{n-1} p_{T *}^{\prime}((3.9))$ induces

$$
\phi: R^{n-1} p_{T *} \operatorname{Hom}\left(F^{*} \mathcal{L}^{\prime}, \omega\right) \rightarrow R^{n-1} p_{T *}^{\prime} \operatorname{Hom}\left(\mathcal{L}^{\prime}, \omega^{\prime}\right) .
$$

For any $t \rightarrow T$, let $X_{t}$ be the fiber of $X_{T} \rightarrow T$, and $F_{t}: X_{t} \rightarrow X_{t}^{\prime}$ be the relative Frobenius. Then $(3.10) \otimes k(t)$ induces (through $\tau$ )

$$
\phi \otimes k(t): H^{n-1}\left(X_{t}, \operatorname{Hom}\left(F_{t}^{*} \mathcal{L}_{t}^{\prime}, \omega_{t}\right)\right) \rightarrow H^{n-1}\left(X_{t}^{\prime}, \operatorname{Hom}\left(\mathcal{L}_{t}^{\prime}, \omega_{t}^{\prime}\right)\right)
$$

which is induced by $\varphi:=(3.9) \otimes k(t)$. Then the surjection of vector bundles on $X_{t}^{\prime}$

$$
\varphi:\left(F_{t}\right)_{*} \operatorname{Hom}\left(F_{t}^{*} \mathcal{L}_{t}^{\prime}, \omega_{t}\right) \rightarrow \operatorname{Hom}\left(\mathcal{L}_{t}^{\prime}, \omega_{t}^{\prime}\right)
$$

is dual, by taking $\operatorname{Hom}\left(\cdot, \omega_{t}^{\prime}\right)$, to the canonical injection

$$
\mathcal{L}_{t}^{\prime} \hookrightarrow\left(F_{t}\right)_{*} F_{t}^{*} \mathcal{L}_{t}^{\prime}
$$

of vector bundles on $X_{t}^{\prime}$. As the dual $H^{n-1}(\varphi)^{\vee}$ of $H^{n-1}(\varphi)=\phi \otimes k(t)$ is the $k(t)$-linear map

$$
F_{t}^{*}: H^{1}\left(X_{t}^{\prime}, \mathcal{L}_{t}^{\prime}\right) \rightarrow H^{1}\left(X_{t}, F_{t}^{*} \mathcal{L}_{t}^{\prime}\right)
$$

induced by (3.12), this finishes the proof.

To prove Theorem 3.4, the key tool is a theorem of Hrushovski. In fact, we only need a special case of his theorem:

Theorem 3.7 (Hrushovski, [6, Corollary 1.2]). Let $Y$ be an affine variety over $\mathbb{F}_{q}$, and let $\Gamma \subset\left(Y \times_{\mathbb{F}_{q}} Y\right) \otimes_{\mathbb{F}_{q}} \overline{\mathbb{F}}_{q}$ be an irreducible subvariety over $\overline{\mathbb{F}}_{q}$. Assume the two projections $\Gamma \rightarrow Y$ are dominant. Then, for any closed subvariety $W \varsubsetneqq Y$, there exists $x \in Y\left(\overline{\mathbb{F}}_{q}\right)$ such that $\left(x, x^{q^{m}}\right) \in \Gamma$ and $x \notin W$ for large enough natural number $m$.

Remark 3.8. Writing $Y \subset \mathbb{A}^{r}$, we have $x=\left(x_{1}, \ldots, x_{r}\right) \in\left(\overline{\mathbb{F}}_{q}\right)^{r}=\mathbb{A}^{r}\left(\overline{\mathbb{F}}_{q}\right)$ and $x^{q^{m}}:=\left(x_{1}^{q^{m}}, \ldots, x_{r}^{q^{m}}\right) \in \mathbb{A}^{r}\left(\overline{\mathbb{F}}_{q}\right)$. In our application, $\Gamma$ is the Zariski closure of the graph of a dominant rational map $f: Y \rightarrow Y$ and $W$ is the locus where $f$ is not well-defined.

Proof of Theorem 3.4. (Compare with [3, Theorem 3.14].) Let

$$
f: M \rightarrow M
$$

be as in Proposition 3.5. So $M \rightarrow S$ is an $S$-reduced scheme of finite type, where $S$ is smooth affine over $\mathbb{F}_{q}$, the normalization of $\mathbb{F}_{p}$ in $H^{0}\left(S, \mathcal{O}_{S}\right)$. Let $M_{k}$ be the 
general fiber of $M \rightarrow S$ at $\operatorname{Spec}(k) \rightarrow S$. We define $Z_{k}$ to be the intersection of the Zariski closures $\overline{\mathbb{E}_{X}(m)}$ of the $\mathbb{E}_{X}(m) \subset M_{k}$, where $\mathbb{E}_{X}(m)=\left\{\left(L_{i+m} \hookrightarrow\right.\right.$ $\left.\left.V_{i+m} \rightarrow \mathcal{O}_{X}\right) \in \mathbb{E}_{X}\right\}_{i \in \mathbb{N}}$. By the Nötherian property, there exists a $m_{0}>0$ such that

$$
Z_{k}=\overline{\mathbb{E}_{X}(m)} \subset M_{k}(k)=M(k)
$$

for any $m \geq m_{0}$. As $\mathbb{E}_{X}$ is assumed to be infinite, all components of $Z_{k}$ have dimension $\geq 1$. Indeed, if it had a component of dimension 0 , then there would be a $m_{1}>m_{0}$ such that this 0-dimensional component would not lie on $\mathbb{E}_{X}\left(m_{1}\right)$, a contradiction. The fields of constants of the irreducible components of $Z_{k}$ lie between $\mathbb{F}_{q}(S)$ and $k$. Let $S^{\prime} \rightarrow S$ be affine with $S^{\prime}$ smooth affine irreducible such that all components of $Z_{k}$ are defined over $S^{\prime}$, yielding models of them over $S^{\prime}$ which are geometrically irreducible over $\mathbb{F}_{q}$, and a model $Z \rightarrow S^{\prime}$ of $Z_{k}$. We replace now $X_{S} \rightarrow S$ and $M \rightarrow S$ by $X_{S^{\prime}}$ and the corresponding $M_{S^{\prime}} \rightarrow S^{\prime}$. We abuse notations, set $S=S^{\prime}$, and have $Z \subset M \rightarrow S$.

Let $M^{0} \subset M$ be the largest open subset where $f$ is well-defined. Notice that

$$
\mathbb{E}_{X}(1) \subset M_{k}^{0}
$$

and $f\left(\mathbb{E}_{X}(m)\right)=\mathbb{E}_{X}(m-1)$ for any $m \geq 1$. Thus $f\left(Z_{k} \cap M_{k}^{0}\right)$ contains a dense subset of $Z_{k}$. On the other hand,

$$
f\left(Z_{k} \cap M_{k}^{0}\right) \subset Z_{k}
$$

thus $f: M \rightarrow M$ induces a dominant rational map $f: Z_{k} \rightarrow Z_{k}$, and thus a rational dominant map

$$
f: Z \rightarrow-\rightarrow \text {. }
$$

We consider one irreducible component of $Z_{k}$, and its model $Z^{\text {irr }} \rightarrow S$, which is a geometrically irreducible component of $Z$ over $\mathbb{F}_{q}$. There is an integer $a_{1}>0$ such that $f^{a_{1}}: Z^{\text {irr }} \rightarrow-Z^{\text {irr }}$ is a dominant rational map over a finite field $\mathbb{F}_{q}$.

To prove that $Z^{\text {irr }}$ contains a periodic point of $f^{a_{1}}$, without loss of generality, we can assume that $Y:=Z^{\text {irr }} \subset \mathbb{A}^{r}$ is an affine variety and $f^{a_{1}}: Y \rightarrow Y$ is defined by rational functions $f_{1}, \ldots, f_{r} \in \mathbb{F}_{q}(Y)$. Let

$$
\Gamma \subset Y \times \mathbb{F}_{q} Y
$$

be the Zariski closure of the graph of $f^{a_{1}}$, which is irreducible over $\overline{\mathbb{F}}_{q}$ as $Y$ is by assumption. The two projections $\Gamma \rightarrow Y$ are dominant since $f^{a_{1}}: Y \rightarrow Y$ is dominant. By Theorem 3.7, there exists a point $x \in Y\left(\overline{\mathbb{F}}_{q}\right)$ such that $f$ is welldefined at $x=\left(x_{1}, \ldots, x_{r}\right)$ and $f^{a_{1}}(x)=\left(x_{1}^{q^{m}}, \ldots, x_{r}^{q^{m}}\right):=x^{q^{m}}$ for some large $m$. Recall that $f_{i} \in \mathbb{F}_{q}(Y)(i=1, \ldots, r)$ have coefficients in $\mathbb{F}_{q}$, we have

$$
\begin{aligned}
f^{a_{1}}\left(f^{a_{1}}(x)\right) & =\left(f_{1}\left(x_{1}^{q^{m}}, \ldots, x_{r}^{q^{m}}\right), \ldots, f_{1}\left(x_{1}^{q^{m}}, \ldots, x_{r}^{q^{m}}\right)\right) \\
& =\left(f_{1}\left(x_{1}, \ldots, x_{r}\right)^{q^{m}}, \ldots, f_{1}\left(x_{1}, \ldots, x_{r}\right)^{q^{m}}\right) \\
& =\left(x_{1}^{q^{2 m}}, \ldots, x_{r}^{q^{2 m}}\right)=x^{q^{2 m}} .
\end{aligned}
$$


Thus $f^{a_{2} a_{1}}(x)=x^{q^{a_{2} m}}=x$ when $a_{2}$ is large enough. The point

$$
x \in Y\left(\overline{\mathbb{F}}_{q}\right)
$$

determines a nontrivial extension $0 \rightarrow L \rightarrow V \rightarrow \mathcal{O}_{X_{\bar{s}}} \rightarrow 0$ on a good reduction $X_{\bar{s}}$ of $X$ (over $\overline{\mathbb{F}}_{q}$ ). That $f^{a}(x)=x, a=a_{1} a_{2}$, means by Proposition 3.5 (3)

$$
\left(\left(F_{X_{\bar{s}}}^{*}\right)^{a} L \hookrightarrow\left(F_{X_{\bar{s}}}^{*}\right)^{a} V \rightarrow\left(F_{X_{\bar{s}}^{*}}^{*}\right)^{*} \mathcal{O}_{X_{\bar{s}}}\right) \cong\left(L \hookrightarrow V \rightarrow \mathcal{O}_{X_{\bar{s}}}\right) .
$$

This finishes the proof of Theorem 3.4 and thus of Theorem 3.1.

Theorem 3.9. Let $X$ be a smooth projective variety over an algebraically closed field $k$ of characteristic $p>0$. Then:

(i) Every stratified bundle on $X$ is trivial if and only if $\pi_{1}$ is trivial;

(ii) All the irreducible stratified bundles have rank 1 if and only if $\left[\pi_{1}, \pi_{1}\right]$ is a pro-p-group;

(iii) Every stratified bundle is a direct sum of stratified line bundles, that is $\operatorname{str}(X)$ is a semi-simple category with irreducible objects of rank 1 , if and only if $\pi_{1}$ is Abelian with no non-trivial p-power quotient.

Proof. (i) is the main theorem of [3]. (ii) is Theorem 2.6. To show (iii), assume that $\pi_{1}$ is Abelian with no $p$-power order quotient. Let $E=\left(E_{n}, \sigma_{n}\right)_{n \in \mathbb{N}}$ be a stratified bundle on $X$. By (ii), any irreducible stratified bundle has rank 1 . Thus there is a filtration $0=E^{0} \subset E^{1} \subset \cdots \subset E^{r}=E$ in $\operatorname{str}(X)$ such that $L^{v}=E^{v} / E^{v-1}$, $1 \leq v \leq r$, are rank one stratified bundles. Then, by Theorem 3.1, the filtration splits and $E$ is a direct sum of rank-1 objects.

We now comment on analogues of (ii) and (iii) in complex geometry. For this reason, we include here the following lemma, which may have an independent interest.

Lemma 3.10. Let $G$ be a commutative group scheme over an algebraically closed field $k$ such that all its quotients in $\mathbb{G}_{m}$ are smooth. Let $\ell$ be a non-trivial character of $G$. Then $H^{1}(G, \ell)=0$.

Proof. Let $\chi: G \rightarrow \mathbb{G}_{m}=\operatorname{Aut}(\ell)$ be the non-trivial character, and let $\sigma: G \rightarrow \ell$ be a cocycle representing a class in $H^{1}(G, \ell)$. By definition of a cocycle, one has $\sigma(g h)=\chi(g) \sigma(h)+\sigma(g)$. The commutativity of $G$ implies

$$
\sigma(h g)=\chi(h) \sigma(g)+\sigma(h)=\sigma(g h)=\chi(g) \sigma(h)+\sigma(g) .
$$

As $\chi$ is non-trivial, and $\operatorname{Im}(\chi) \subset \mathbb{G}_{m}$ is assumed to be smooth, there is a $h \in G(k)$ such that

$$
0 \neq(\chi(h)-1) \in \operatorname{End}(\ell)=k .
$$

Thus

$$
(\chi(h)-1) \in k^{\times}=\mathbb{G}_{m}(k)=\operatorname{Aut}(\ell) .
$$

Set $v=(\chi(h)-1)^{-1} \sigma(h) \in \ell$. Then, by (3.2), one has $\sigma(g)=\chi(g) v-v$, which means that $\sigma$ is a coboundary. Thus $H^{1}(G, \ell)=0$. 
Remark 3.11. The same proof shows that if $X$ is a smooth complex variety, with Abelian topological fundamental group, and if $\ell$ is a non-trivial rank-1 local system, then $H^{1}(X, \ell)=0$. Indeed, $G$ is now $\pi_{1}^{\text {top }}(X, a)$, the topological fundamental group based at a complex point $a \in X(\mathbb{C})$, and non-triviality implies the existence of $h \in G$ with $(\chi(h)-1) \in \mathbb{C}^{\times}=\operatorname{Aut}(\ell)$. One then concludes identically.

This fact ought to be well known, but we could not find a reference in the literature.

Remark 3.12. As already mentioned in the introduction, the Malcev-Grothendieck theorem $([5,10])$ asserts that the étale fundamental group $\pi_{1}$ of a smooth complex projective variety is trivial if and only its stratified fundamental group $\pi^{\text {str }}$ is trivial, where $\pi^{\text {str }}$ is the pro-affine complex algebraic group of the Zariski closures of the monodromies of complex linear representations of the topological fundamental group $\pi_{1}^{\text {top }}$. By going to the associated Galois cover, it implies that $\pi^{\text {str }}$ is finite if and only if $\pi_{1}$ is finite (in which case $\pi^{\text {str }}=\pi_{1}$ ). One also has that $\pi^{\text {str }}$ is Abelian if and only if $\pi_{1}$ is Abelian. Indeed, by definition $\pi^{\text {str }}$ is Abelian if and only irreducible complex linear representations have dimension 1 . But if $\rho: \pi_{1}^{\text {top }} \rightarrow$ $G L(r, \mathbb{C})$ is a representation, since $\pi_{1}^{\text {top }}$ is spanned by finitely many elements, $\rho$ has values in $G L(r, A)$ for $A$ a ring of finite type over $\mathbb{Z}$. Then if $\rho$ is irreducible, there is a closed point $s \in \operatorname{Spec}(A)$ such that $\rho \otimes \kappa(s): \pi_{1}^{\text {top }} \rightarrow G L(r, \kappa(s))$ is irreducible as well, where $\kappa(s)$ is the residue field of $s$. As $\rho \otimes \kappa(s)$ is finite, it factors through $\pi_{1}$, thus $r=1$. Since a linear representation with finite monodromy is semi-simple, one can summarize as follows:

Claim 3.13. $\pi_{1}$ is

1) finite,

2) respectively, Abelian,

3) respectively, Abelian and finite,

if and only if $\pi_{1}^{\text {str }}$ is

1) finite,

2) respectively, Abelian,

3) respectively, Abelian and finite,

if and only if

1) local systems have finite monodromy,

2) respectively, irreducible local systems have rank-1,

3) respectively, local systems are direct sums of rank-1 local systems.

While the main result of [3] is an analogue in characteristic $p>0$ of Claim 3.13 (1), the main theorem of this note is an analogue of Claim 3.13 (2) and (3). 


\section{References}

[1] B. ConRaD, "Grothendieck Duality and Base Change", Lecture Notes in Mathematics, Vol. 1750, Springer, Berlin, 2000.

[2] J. P. P. DOs S ANTOS, Fundamental group schemes for stratified sheaves, J. Algebra 317 (2007), 691-713.

[3] H. Esnault and V. Menta, Simply connected projective manifolds in characteristic $p>0$ have no nontrivial stratified bundles, Invent. Math. 181 (2010), 449-465.

[4] D. GIESEKER, Flat vector bundles and the fundamental group in non-zero characteristics, Ann. Scuola Norm. Sup. Pisa Cl. Sci. (2) 4 (1975), 1-31.

[5] A. GROTHENDIECK, Représentations linéaires et compactifications profinies des groupes discrets, Manuscripta Math. 2 (1970), 375-396.

[6] E. HRUShOVSKI, The elementary theory of the Frobenius automorphism, arXiv: math/0406514v1 [math.LO], 2004.

[7] D. Huybrechts and M. LEHN, "The Geometry of the Moduli Space of Sheaves", Aspects of Mathematics, Vol. E31, Vieweg \& Sohn, Braunschweig, 1997.

[8] S. Kleiman, The Picard scheme, In: "Fundamental Algebraic Geometry", Math. Surveys Monogr. 123, American Mathematical Society, Providence, R.I., 2005, 235-321.

[9] H. LANGE and U. STUhLER, Vektorbündel auf Kurven und Darstellungen der Algebraischen Fundamentalgruppe, Math. Z. 156 (1977), 73-83.

[10] A. MALCEV, On isomorphic matrix representations of infinite groups, Rec. Math. [Mat. Sbornik] N.S. 8 (50) (1940), 405-422.

[11] D. Mumford, "Abelian Varieties", Tata Institute of Fundamental Research Studies in Mathematics 5, Oxford University Press, London, 1970.

[12] J.-P. SERRE, "Linear Representations of Finite Groups", Graduate Texts in Mathematics, Vol. 42, 1977.

[13] A. GrothendiECK, "Revêtements étales et groupe fondamental", Séminaire de Géométrie Algébrique SGA 1, Lecture Notes in Mathematics, Vol. 224, Springer-Verlag, Berlin (1971).

Freie Universität Berlin

Arnimallee, 3

14195 Berlin, Germany

esnault@math.fu-berlin.de

Academy of Mathematics and Systems Science

Chinese Academy of Science

Beijing, P. R. of China

xsun@math.ac.cn 